\title{
PRR11 and SKA2 gene pair is overexpressed and regulated by p53 in breast cancer
}

\author{
Yitao Wang ${ }^{1}$, Chunxue Zhang ${ }^{2}$, Li Mai ${ }^{3}$, Yulong Niü ${ }^{1}$, Yingxiong Wang $^{4}$ \& Youquan Bu ${ }^{1, *}$ \\ ${ }^{1}$ Department of Biochemistry and Molecular Biology, Chongqing Medical University, Chongqing 400016, ${ }^{2}$ Laboratory of Birth Defects and \\ Related Diseases of Women and Children, West China Second University Hospital, Sichuan University, Chengdu 610000, ${ }^{3}$ Department of \\ Clinical Laboratory, The Second Affiliated Hospital, Chongqing Medical University, Chongqing 400010, ${ }^{4}$ Laboratory of Reproductive \\ Biology, School of Public Health, Chongqing Medical University, Chongqing 400016, China
}

Our previous study found that two novel cancer-related genes, PRR11 and SKA2, constituted a classic gene pair that was regulated by p53 and NF-Y in lung cancer. However, their role and regulatory mechanism in breast cancer remain elusive. In this study, we found that the expression levels of PRR11 and SKA2 were upregulated and have a negative prognotic value in breast cancer. Loss-of-function experiments showed that RNAi-mediated knockdown of PRR11 and/or SKA2 inhibited proliferation, migration, and invasion of breast cancer cells. Mechanistic experiments revealed that knockdown of PRR11 and/or SKA2 caused dysregulation of several downstream genes, including CDK6, TPM3, and USP12, etc. Luciferase reporter assays demonstrated that wild type p53 significantly repressed the PRR11-SKA2 bidirectional promoter activity, but not NF-Y. Interestingly, NF-Y was only essential for and correlated with the expression of PRR11, but not SKA2. Consistently, adriamycin-induced (ADR) activation of endogenous p53 also caused significant repression of the PRR11 and SKA2 gene pair expression. Notably, breast cancer patients with lower expression levels of either PRR11 or SKA2, along with wild type p53, exhibited better disease-free survival compared to others with p53 mutations and/or higher expression levels of either PRR11 or SKA2. Collectively, our study indicates that the PRR11 and SKA2 transcription unit might be an oncogenic contributor and might serve as a novel diagnostic and therapeutic target in breast cancer. [BMB Reports 2019; 52(2): 157-162]

*Corresponding author. Tel: +86-23-68485991; Fax: +86-23-6848 5991; E-mail: buyqcn@cqmu.edu.cn

https://doi.org/10.5483/BMBRep.2019.52.2.207

Received 7 September 2018, Revised 2 November 2018, Accepted 31 December 2018

Keywords: Breast cancer, p53, PRR11, SKA2

\section{INTRODUCTION}

Breast cancer is one of the most common causes of cancer deaths in women. The five-year survival rate of breast cancer patients in China is $73.1 \%$, and in the United States it is 89.4 percent $(1,2)$. One of the primary reasons for the lower survival in China is the low early screening rate for breast cancer. Finding and identifying novel breast cancer-related genes will assist in early screening and treatment of breast cancer.

More than $10 \%$ of the genes in the human genome are closely spaced, sharing a bidirectional promoter (3). Two adjacent genes that can be regulated by a single bi-directional promoter are defined as a gene pair. Our previous study found that the proline-rich 11 (PRR11) and the spindle and kinetochore-associated complex subunit 2 (SKA2) genes share a typical bidirectional promoter, and are regulated by $\mathrm{p} 53$ and nuclear transcription factor $\mathrm{Y}$ (NF-Y) in lung cancer. PRR11 is cell cycle-dependent, closely related to the occurrence and development of various tumors, and is highly-expressed in lung cancer, hilar cholangiocarcinoma, and gastric cancer cells. Knockdown of PRR11 can lead to cell cycle arrest and inhibition of cell proliferation, migration, and invasion (4-6). In addition, overexpression of PRR11 in $\mathrm{H} 1299$ cells led to precocious chromosome agglutination and apoptosis, suggesting that PRR11 plays a role in lung cancer by affecting chromosomal stability (7).

SKA2 participates in the formation of Ska complex and plays an important role in the maintenance of the mitotic mid-plateau and shut-down of the spindle checkpoint (8-14). SKA2 can also participate in cell proliferation and tumorigenesis by interacting with glucocorticoid receptors (15). Current studies on PRR11 and SKA2 have mostly concentrated on lung cancer, and studies on their effects on breast cancer are relatively rare. Research on the mechanism of the gene pair PRR11 and SKA2 expression and regulation is still in its early stages and further studies are needed.

This study analyzed the clinical significance of the gene pair PRR11 and SKA2 in breast cancer, and its effect on the proliferation, migration, and invasion of breast cancer cell

ISSN: 1976-670X (electronic edition)

Copyright (C) 2019 by the The Korean Society for Biochemistry and Molecular Biology

(ㄷ) This is an open-access article distributed under the terms of the Creative Commons Attribution Non-Commercial License (http://creativecommons.org/licenses/by-nc/4.0) which permits unrestricted non-commercial use, distribution, and reproduction in any medium, provided the original work is properly cited. 
lines by RNA interference. We also investigated whether the transcription factors, NF-Y and p53, regulate the PRR11-SKA2 gene pair expression. In conclusion, our study further explored the role of the gene pair PRR11 and SKA2 in breast cancer and laid a foundation for its molecular mechanism.

\section{RESULTS}

\section{PRR11 and SKA2 are upregulated in breast cancer tissues}

To analyze the expression of PRR11 and SKA2 in breast cancer tissues, we downloaded a group of RNA-sequence data from the cancer genome atlas (TCGA). Expression of PRR11 and SKA2 mRNA were higher in cancer tissues than in the normal tissue (Fig. 1A). And their expression was higher in cancer tissues than in para-cancer tissues (Fig. 1B). Next, we analyzed the prognostic value of PRR11 and SKA2 in breast cancer. First, the GSE3494 data set showed that high expression levels of PRR11 and/or SKA2 was correlated with shorter disease-free survival of breast cancer patients (Fig. 1C). Second, we used the composite Kaplan-Meier (KM) Plotter to analyze the

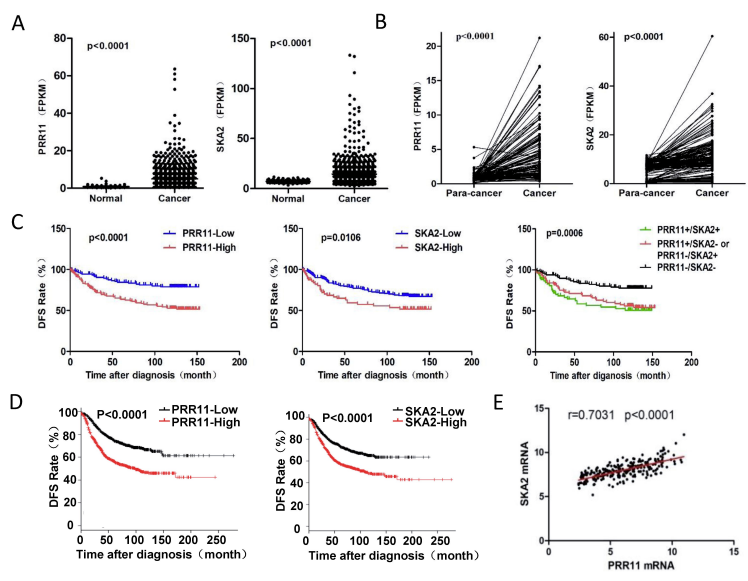

Fig. 1. PRR 11 and $S K A 2$ are upregulated in breast cancer, and their expression correlated with disease-free survival. (A) Expression of PRR11 and SKA2 mRNA in breast cancers. PRR11 and SKA2 expression levels in normal and cancerous breast tissues were analyzed using the TCGA and GEO data set (GSE3494). Differences in PRR11 and SKA2 mRNA levels between two independent cohorts were assessed by the non-parametric Mann-Whitney $U$ test. (B) Expression of PRR11 and SKA2 was higher in breast cancer tissues than that in para-cancer tissues. (C) and (D) Disease-free survival analysis in relation to PRR11 and SKA2 expression in breast cancer. Kaplan-Meier plots of disease-free survival of breast cancer patients in the GSE3494 data set (C). Additional data sets from GEO and TCGA were used to determine the clinical significance using the Kaplan-Meier Plotter online tool. These data sets included GSE7390, GSE41998, GSE26971, GSE21653, GSE20685, GSE2034, and GSE12276 (D). (E) Correlation between mRNA expression of PRR11 and SKA2 was determined in GSE3494 by Spearman statistical analysis. The $\mathrm{Y}$ - and $\mathrm{X}$-axes indicate the relative mRNA expression levels of PRR11 and SKA2, respectively. prognosis of women with PRR11 and SKA2, and the result correlated with the results of the GSE3494 data set (Fig. 1D). In addition, PRR11 levels closely correlated with SKA2 levels in breast cancer patients (Fig. 1E).

\section{Knockdown of PRR11 and SKA2 inhibits breast cancer cell proliferation, migration, and invasion}

Our previous study showed that knockdown of PRR11 and/or SKA2 resulted in a significant decrease in cell proliferation, migration, and invasion in lung cancer cells (16). To identify the function of the gene pair PRR11and SKA2 in breast cancer cells, we used specific siRNA targeting PRR11 and/or SKA2 in MCF7 and MDA-MB-231 cells. QPCR and Western blotting analyses showed that we successfully knocked-down PRR11 and/or SKA2 (Fig. 2A, B, D, E). The cell counting kit-8 (CCK-8) assay showed that knockdown of PRR11 and/or SKA2 decreased breast cancer cell proliferation (Fig. 2C, F). Then, we investigated whether decreased expression of the gene pair affected cell migration and invasion in breast cancer cells. Knockdown of PRR11 and/or SKA2 significantly decreased the
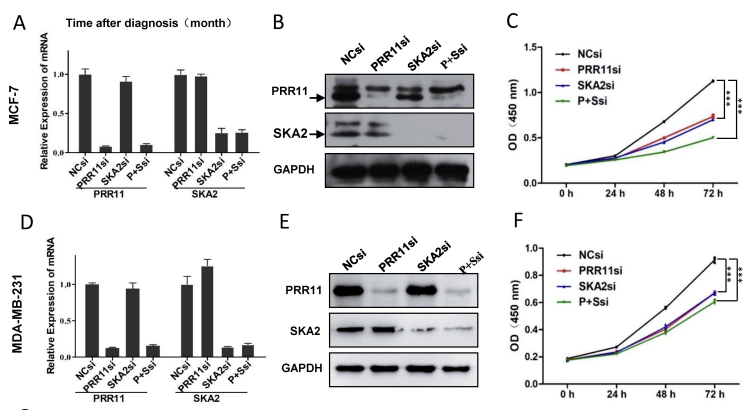

G
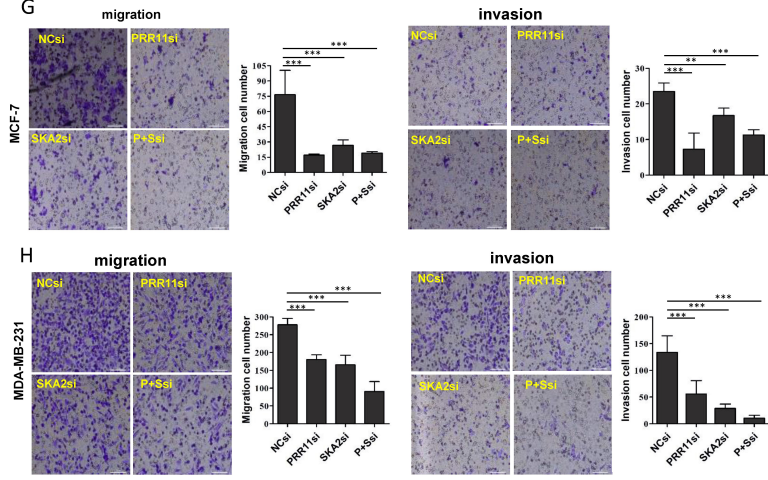

Fig. 2. Silencing of PRR11 and SKA2 represses proliferation, migration, and invasion of breast cancer cells. MCF7 and MDA-MB-231 cells were transiently transfected with siRNA against PRR11 and/or SKA2. Forty-eight hours after transfection, whole-cell lysates were prepared and subjected to QPCR analysis (A and D), and immunoblotted ( $B$ and $E$ ). Cells transfected as above were also subjected to the proliferation assay ( $\mathrm{C}$ and $\mathrm{F}$ ), trans-well migration, and invasion assays $(G$ and $H)$. Student's t tests were used to compare the data from three PRR11 and/or SKA2 silencing groups with that from the NCsi group $(* * * P<0.001)$. 
cellular migration and invasion of both MCF7 and MDA-MB-231 cells (Fig. 2G, H). Taken together, our results indicated that PRR11 and/or SKA2 expression was essential for maintaining the accelerated proliferation, migration, and invasive capabilities of breast cancer cells.

\section{p53 inhibits transactivation of the PRR11 and SKA2 gene pair in breast cancer cells}

Our previous study indicated that the PRR11-SKA2 bidirectional transcription unit was negatively regulated by p53 through NF-Y in lung cancer cells. To investigate whether p53 could regulate the gene pair in breast cancer in the same way, MCF7 cells were co-transfected with p53 or the nuclear transcription factor $\mathrm{Y}$ subunit beta (NFYB) expression vector and a series of PRR11-SKA2 luciferase reporters. The luciferase results showed that $\mathrm{p} 53$, but not NFYB, caused a decrease in luciferase activity of PRR11- and SKA2-orientated promoter reporter deletion constructs (Fig. 3). Thus, these results showed that p53 repressed the PRR11-SKA2 bidirectional promoter in both PRR11 and SKA2 orientations in breast cancer, but that NFYB did not transactivate the PRR11-SKA2 bidirectional promoter.

\section{p53 inhibits the expression of PRR11 and SKA2 gene pair and its clinical significance in breast cancer}

Next, we investigated whether p53 inhibited the expression of PRR11 and SKA2 in breast cancer. ADR was used to treat p53

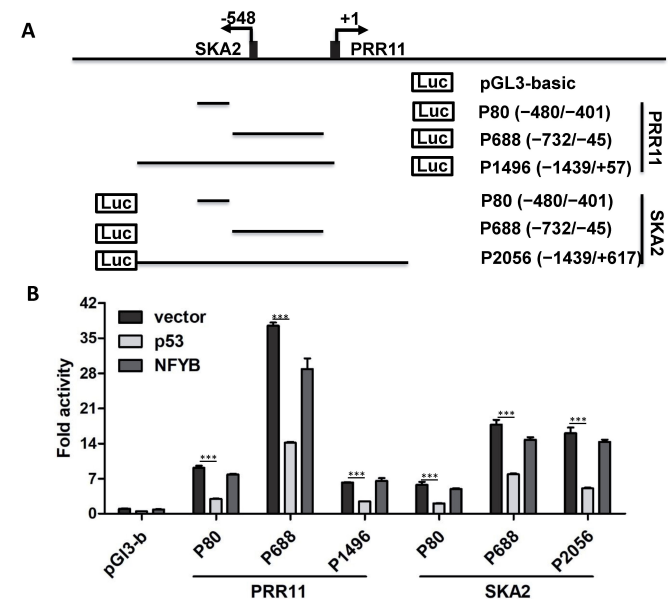

Fig. 3. p53 represses PRR11 and SKA2 bidirectional promoter Overexpression of wild-type p53 inhibited the activity of the PRR11 and SKA2 bidirectional promoter. MCF7 cells were transiently co-transfected with the indicated luciferase reporter constructs and pRL-TK, together with the expression plasmid for wild-type p53. Forty-eight hours after transfection, their luciferase activities were examined by the Dual Luciferase Assay System (Promega). Data obtained from a representative of at least three independent experiments are shown as fold-induction compared to the activity of cells transfected with the empty pGL3-basic vector. Two independent experiments were performed in triplicate $(* * * P<0.001)$. wild-type MCF7 cells. QPCR and Western blotting analyses indicated that ADR induced p53 activation and repression of the endogenous PRR11 and SKA2 expression at both mRNA and protein levels (Fig. 4A, B). Since p53 is often mutated in cancer cells, we used the GSE3494 data set to analyze the expression of PRR11 and SKA2 in p53-mutated and wild-type breast cancer patients. The results showed that the expression of PRR11 and SKA2 was higher in p53-mutated breast cancer than in p53 wild-type (Fig. 4C, D). To ask whether p53-mediated PRR11-SKA2 repression represented clinical significance in breast cancer, we examined the prognostic value of PRR11 and SKA2 with different p53 statuses in the GSE3494 data set in which the p53 status of each patient was available. Univariate survival analysis showed that breast cancer patients with wild-type p53, along with lower expression of either PRR11 or SKA2, exhibited the best disease-free survival compared to those with p53 mutations and/or higher expression of either PRR11 or SKA2 (Fig. 4E). These results indicate that the wild type p53 inhibited the expression of PRR11 and SKA2 and that repression of the gene pair showed the best survival for breast cancer patients.

A

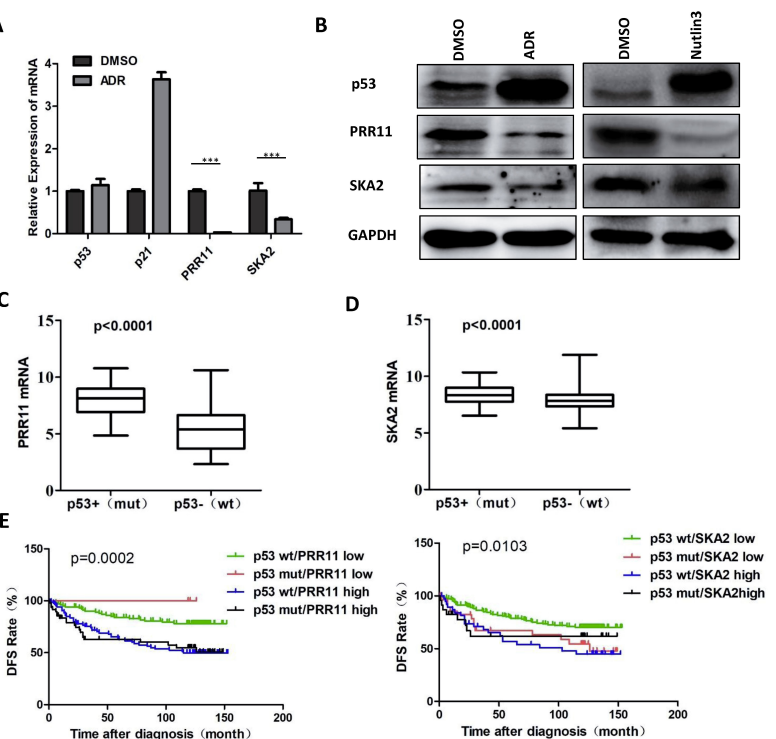

Fig. 4. PRR 11 and SKA2 are regulated by DNA damage-induced p53. MCF7 cells was exposed to $1 \mu \mathrm{M}$ of ADR or left untreated. Thirty-six hours after treatment, cells were subjected to qPCR analysis (two-sided independent Student's $\mathrm{t}$ test, $* * * \mathrm{P}<0.001$ ) (A); immunoblot analysis (B); (C) and (D) mRNA expression levels of PRR11 and SKA2 were analyzed in patients with wild-type p53 or mutant p53 in the GEO data set (GSE3494); (E) and analysis of disease-free breast cancer survival according to PRR11 and SKA2 expression with different p53 statuses. Kaplan-Meier plots of disease-free survival of breast cancer patients in the GEO data set (GSE3494). 


\section{DISCUSSION}

Genes regulated by bidirectional promoters can be divided into multiple groups according to their function, related not only to DNA repair-related pathways, but also to the maintenance of genomic stability, and other life activities. More and more evidence has shown that bidirectional promoters were closely related to the development of multiple cancers. Our previous study found that the gene pair PRR11 and SKA2 was regulated by NF-Y and p53 in lung cancer and contributed to lung cancer development (16-18). In the present study, we found that the gene pair PRR11 and SKA2 was negatively regulated by p53, but not by NF-Y, and was essential for the accelerated proliferation and motility of breast cancer cells.

Our previous study focused on the function of the gene pair PRR11 and SKA2 in lung cancer. When we analyzed the expression and prognosis of the gene pair in breast cancer by GEO and TCGA data sets, we found that the expression of the gene pair was higher in breast cancer tissues than in normal tissues, and was highly correlated with the expression of PRR11 and SKA2 (Fig. 1A, B, E). Moreover, further analysis revealed that high expression of the gene pair in breast cancer was significantly associated with poorer patient disease-free survival (Fig. 1C, D), suggesting that the gene pair might be a novel diagnostic and gene therapy target. Other studies have revealed that PRR11 was elevated in hilar cholangiocarcinoma and gastric cancer, but there have been no reports on the function and mechanisms of the gene pair in breast cancer (5, $6,19)$.

Herein, our results showed that PRR11 and SKA2 silencing alone or in combination inhibited cell proliferation, migration, and invasion in MCF-7 and MDA-MB-231 breast cancer cells (Fig. 2). Under the condition of combined silencing, the inhibition was stronger, so we speculated that PRR11 and SKA2 could work together in some respects. Further study revealed that when PRR11 and SKA2 were silenced alone or in combination, the expression of multiple genes related to cell proliferation, migration, and invasion, such as CDK6, IL6ST, RBMS1, TPM3, USP12, and CASP7, were significantly changed (data are not shown) (20-26).

Our previous studies found that the gene pair PRR11 and SKA2 was positively regulated by the transcription factor NF-Y in lung cancer cells. That is, NF-Y promoted the expression of the gene pair, and the p53 transcription factor inhibited the expression of PRR11 and SKA2 indirectly through NF-Y (16). However, this study found that in breast cancer cells, NF-YB silencing inhibited the expression of PRR11, but not of SKA2. Further analysis found that the expression level of NFYB in breast cancer correlated with low PRR11 expression, but not with SKA2 expression. And NF-Y did not activate the bidirectional promoter of the gene pair PRR11 and SKA2 (Fig. 3B). This result suggests that NF-Y had a preference for the regulation of the gene pair PRR11 and SKA2 in breast cancer cells and that it may be affected by different cell types.

p53 is an important tumor suppressor gene and transcription regulatory factor. It plays an important role in maintaining the stability of the genome and in inhibiting the development of tumors by participating in major life activities, such as cell cycle regulation, DNA damage repair, apoptosis, and aging $(26,27)$. The function of p53 can be controlled indirectly through direct binding to the promoter of the target gene or by interaction with other transcription factors (e.g., NF-Y, Sp1, etc.), and thereby indirectly regulate gene expression. Our study found that p53 inhibited the expression of the gene pair PRR11 and SKA2 in MCF7 breast cancer cells (Fig. 4A, B). In addition, the expression of PRR11 and SKA2 was higher in p53-mutated patients than in wild type (Fig. 4C, D). Breast cancer patients with mutated p53 and high expression of PRR11 or SKA2 expression had worse prognoses than the other groups (Fig. 4E). So, it is possible that p53 indirectly regulated the expression of the gene pair by interacting with other transcription factors, however, the identification and mechanisms of related transcription factors still require further exploration.

In summary, the gene pair PRR11 and SKA2 played an important role in the development of breast cancer. More and more evidence has shown that the PRR11 and SKA2 gene pair is a potential oncogenic and prognostic marker. Therefore, it is critically necessary to intensively study the function and mechanism of PRR11-SKA2 for use in breast cancer diagnosis and gene therapy.

\section{MATERIALS AND METHODS}

\section{Cell lines}

MDA-MB-231 and MCF7 cells were cultured in a cell culture incubator $\left(37^{\circ} \mathrm{C}, 5 \% \quad \mathrm{CO}_{2}\right)$ in RPMI 1640 medium (MDA-MB-231) or DMEM (MCF7) supplemented with 100 $\mathrm{mg} / \mathrm{ml}$ streptomycin, 100 units $/ \mathrm{ml}$ penicillin, and $10 \%$ (vol/vol) FBS (Invitrogen). In addition, DMEM (MCF7) was supplemented with $0.01 \mathrm{mg} / \mathrm{ml}$ human recombinant insulin (Sigma, Germany).

\section{siRNA transfection}

siRNAs were transfected into MCF7 and MDA-MB-231 cells using Lipofectamine RNAiMAX reagent (Invitrogen) according to our previous protocol. Cells were subjected to analysis 48 to $72 \mathrm{~h}$ after transfection. Specific siRNAs targeting PRR11 and SKA2 were chemically synthesized by Shanghai GenePharma (Shanghai, China). The sequences of siRNAs used are listed in Supplementary Table S1.

\section{RNA isolation and QRT-PCR}

Total cell RNA isolation and qPCR were conducted as described previously (16). The sequences of primers used are listed in Supplementary Table S1. 


\section{Western blotting}

RIPA buffer supplemented with PMSF was used to lyse cells. Then, total proteins were quantified using the bicinchoninic acid protein assay kit (Thermo Scientific, Beijing, China), and subjected to SDS-PAGE and immunoblotting. The blots were visualized by enhanced chemiluminescence (Bio-Rad Laboratories, Hercules, Ca, USA).

\section{Luciferase reporter assay}

The luciferase reporter constructs (PRR11-P1496, SKA2-P2056, PRR11-P688, SKA2-P688, PRR11-P80, and SKA2-P80) were generated as described previously (16). Cells were seeded onto 12-well plates and co-transfected with the indicated reporter constructs, pRL-TK vector, and the empty vector, or pcDNA3-Flag-p53 or pcDNA3-NFYB expression vectors, using Lipofectamine $^{R} 2000$ reagent (Invitrogen). Luciferase reporter assays were conducted $48 \mathrm{~h}$ after transfection using the Dual-Luciferase assay system (Promega) as described previously (16).

\section{Cell proliferation analysis}

Cells were harvested and 3000 cells/well were seeded onto 96-well plates. The absorbance values at $0,24,48$, and $72 \mathrm{~h}$ were measured using the Cell Counting Kit- 8 . the absorbance (OD) of the corresponding wells was measured at $450 \mathrm{~nm}$. Corresponding measurement values were used as a standard to draw a proliferation histogram.

\section{Cell migration and invasion analysis}

Migration assays were conducted in a 24-well transwell cell culture apparatus fitted with multi-porous polycarbonate membrane inserts (8- $\mu \mathrm{m}$ pore size). Briefly, the top chamber was filled with a cell suspension, and the lower chamber was filled with media supplemented with 10\% FBS. After incubation at $37^{\circ} \mathrm{C}$ in $5 \% \mathrm{CO}_{2}$ for $24 \mathrm{~h}$, the filters were removed, rinsed two times with $\mathrm{PBS}$, fixed with methanol, and stained with $0.5 \%$ crystal violet reagent. Cells on the upper side of the filter were wiped off with cotton swabs. Migrated cells on the lower side of the filter were determined by counting specified cross-sectional fields on the filters (4). Transwell invasion assays were done under the same conditions as the transwell migration assays, but in Matrigel-coated transwells (4).

\section{Prognostic analysis of PRR11 and SKA2 in breast cancer patients}

First, the prognostic value of PRR11 and SKA2 expression was assessed using published breast cancer microarray data from the Uppsala breast cancer database (27). This database includes 249 breast cancer samples. Receiver operating characteristic (ROC) curve analysis was performed to identify a rational cut-off point. Then, we used the Kaplan-Meier Plotter online survival analysis tool to assess the effect of the PRR11 and SKA2 genes on survival using 1764 breast cancer samples (28).

\section{Statistical analyses}

All statistical analyses were carried out using the SPSS 21.0 statistical software package. For gene expression data in breast cancer samples, the Kolmogorov-Smirnov test was used to evaluate the data normality, and either parametric Student's t-tests or nonparametric tests were performed to analyze the significance. Disease-free survival curves were plotted using the Kaplan-Meier method and compared with the log-rank test. Student's t-tests were performed to analyze the other data. P < 0.05 was considered statistically significant.

\section{ACKNOWLEDGEMENTS}

This work was supported by the National Natural Science Foundation of China (No. 81672301 to Youquan Bu), the Basic Sciences and Advanced Technology Key Project of CQ CSTC (No. cstc2017jcyjBX0069 to Youquan Bu), and funded by the Chongqing Special Postdoctoral Science Foundation (No. XmT2018071) and the China Postdoctoral Science Foundation (No. 2018M643422).

\section{CONFLICTS OF INTEREST}

The authors have no conflicting interests.

\section{REFERENCES}

1. Siegel RL, Miller KD and Jemal A (2018) Cancer statistics, 2018: Cancer Statistics, 2018. CA Cancer J Clin 68, 7-30

2. Chen W, Zheng R, Baade PD et al (2016) Cancer statistics in China, 2015: Cancer Statistics in China, 2015. CA Cancer J Clin 66, 115-132

3. Trinklein ND (2003) An Abundance of Bidirectional Promoters in the Human Genome. Genome Res 14 62-66

4. Ji Y, Xie M, Lan H et al (2013) PRR11 is a novel gene implicated in cell cycle progression and lung cancer. Int J Biochem Cell Biol 45, 645-656

5. Song Z, Liu W, Xiao Y et al (2015) PRR11 Is a Prognostic Marker and Potential Oncogene in Patients with Gastric Cancer. PLoS One 10, e0128943

6. Chen Y, Cha Z, Fang W et al (2015) The prognostic potential and oncogenic effects of PRR11 expression in hilar cholangiocarcinoma. Oncotarget 6, 20419-20433

7. Zhang C, Zhang Y, Li Y et al (2015) PRR11 regulates late-S to $\mathrm{G} 2 / \mathrm{M}$ phase progression and induces premature chromatin condensation (PCC). Biochem Biophys Res Commun 458, 501-508

8. Chan YW, Jeyaprakash AA, Nigg EA and Santamaria A (2012) Aurora B controls kinetochore-microtubule attachments by inhibiting Ska complex-KMN network interaction. J Cell Biol 196, 563-571

9. Guimaraes GJ and DeLuca JG (2009) Connecting with Ska, a key complex at the kinetochore-microtubule interface. EMBO J 28, 1375-1377

10. Zhang Q, Sivakumar S, Chen Y et al (2017) Ska3 Phosphorylated by Cdk1 Binds Ndc80 and Recruits Ska to Kinetochores to Promote Mitotic Progression. Curr Biol 27, 
1477-1484.e4

11. Jeyaprakash AA, Santamaria A, Jayachandran $U$ et al (2012) Structural and functional organization of the Ska complex, a key component of the kinetochore-microtubule interface. Mol Cell 46, 274-286

12. Welburn JPI, Grishchuk EL, Backer CB et al (2009) The human kinetochore Ska1 complex facilitates microtubule depolymerization-coupled motility. Dev Cell 16, 374-385

13. Gaitanos TN, Santamaria A, Jeyaprakash AA, Wang B, Conti E, Nigg EA (2009) Stable kinetochore-microtubule interactions depend on the Ska complex and its new component Ska3/C13Orf3. EMBO J 28, 1442-1452

14. Hanisch A, Sillje HH, and Nigg EA (2006) Timely anaphase onset requires a novel spindle and kinetochore complex comprising Ska1 and Ska2. EMBO J 25, 55045515

15. Rice L, Waters CE, Eccles J et al (2008) Identification and functional analysis of SKA2 interaction with the glucocorticoid receptor. J Endocrinol 198, 499-509

16. Wang $Y$, Zhang $Y$, Zhang $C$ et al (2015) The gene pair PRR11 and SKA2 shares a NF-Y-regulated bidirectional promoter and contributes to lung cancer development. Biochim Biophys Acta BBA - Gene Regul Mech 1849, 1133-1144

17. Wang $Y$, Weng $H$, Zhang $Y$ et al (2017) The PRR11-SKA2 bidirectional transcription unit is negatively regulated by p53 through NF-Y in lung cancer cells. Int J Mol Sci 18, 534

18. Zhang L (2018) Silencing of PRR11 suppresses cell proliferation and induces autophagy in NSCLC cells. Genes Dis 5, 158-166

19. Chaudhary S, Madhukrishna B, Adhya AK, Keshari S and Mishra SK (2016) Overexpression of caspase 7 is ERalpha dependent to affect proliferation and cell growth in breast cancer cells by targeting p21(Cip). Oncogenesis 5, e219

20. Murphy CG and Dickler MN (2015) The Role of CDK4/6 inhibition in breast cancer. Oncologist 20, 483-490

21. Wang L, Zhan X, Shen X et al (2018) P16 promotes the growth and mobility potential of breast cancer both in vitro and in vivo: the key role of the activation of IL-6/JAK2/STAT3 signaling. Mol Cell Biochem 446, 137148

22. Selander KS, Li L, Watson L et al (2004) Inhibition of gp130 signaling in breast cancer blocks constitutive activation of Stat 3 and inhibits in vivo malignancy. Cancer Res 64, 6924-6933

23. Niki T, Izumi S, Saëgusa $Y$ et al (2000) MSSP promotes ras/myc cooperative cell transforming activity by binding to c-Myc. Genes Cells Devoted Mol Cell Mech 5, 127141

24. Lange AM and Lo H-W (2018) Inhibiting TRK Proteins in clinical cancer therapy Cancers 10, 105

25. Lehmann BD and Pietenpol JA (2012) Targeting mutant p53 in human tumors. J Clin Oncol Off J Am Soc Clin Oncol 30, 3648-3650

26. Nguyen D, Liao W, Zeng SX and Lu H (2017) Reviving the guardian of the genome: Small molecule activators of p53. Pharmacol Ther 178, 92-108

27. Miller L, Smeds J, George JD et al (2005) An expression signature for p53 status in human breast cancer predicts mutation status, transcriptional effects, and patient survival. Proc Natl Acad Sci U S A 102, 13550-13555

28. Gyorffy B, Lanczky A, Eklund AC et al (2010) An online survival analysis tool to rapidly assess the effect of 22,277 genes on breast cancer prognosis using microarray data of 1,809 patients. Breast Cancer Res Treat 123, 725-731 